\title{
AN INVERSE APPROACH FOR CONSTRUCTING RESIDUAL STRESS FIELD INDUCED BY WELDING
}

\author{
N. $\mathrm{Hu}^{1}$, Y. P. $\mathrm{Cao}^{2},{\text { J. } \mathrm{Lu}^{2} \text { and H. Fukunaga }}^{1}$ \\ 1 Department of Aeronautics and Space Engineering, Tohoku University, Aobayama 01, \\ Aoba-ku, Sendai 980-8579, Japan \\ 2 LASMIS, Universite de technologie Troyes 12, rue Marie Curie-BP 2060-10010 Troyes cedex-France
}

\begin{abstract}
In this paper, an inverse approach based on the inherent strain method is proposed for constructing the residual stress field induced by welding. First, some smooth basis functions in form of polynomial have been employed to approximate the inherent strains. To select the basis functions properly, the previous valuable knowledge about the distributions of residual stress for some typical welding structures should be considered roundly. Furthermore, the singular modes in the assumed inherent strains, which do not cause the residual stresses, are excluded. Then, a stable profile of the inherent strain field can be assumed. Second, by employing the finite element method (FEM) and the least-square technique, the inherent strain field can be identified from limited experimental data at some key points. Finally, the distribution of residual stress can be constructed efficiently using the obtained inherent strain field. Compared with the traditional inherent strain method, in the present work, the sensitivity matrix for predicting the inherent strains can be evaluated more effectively and the experimental data needed in the identification procedure can be reduced significantly. A practical example is used to demonstrate the effectiveness of the present method.
\end{abstract}

\section{INTRODUCTION}

The residual stresses induced in the welding procedure can greatly affect the performance of structures. During the past several decades, a lot of research has been conducted to determine the residual stresses. For instance, various experimental methods and the FEM have been used to determine the residual stresses. In this research, to identify the residual stresses effectively, some regularization techniques have been introduced into the hybrid method by Ueda and Fukuda [1], i.e., the inherent strain method. Comparing with the original method [1], the major advantages of 
the current approach are: 1) the experimental data needed can be reduced dramatically; 2) the singular modes in the inherent strains can be excluded, and then a stable profile of the inherent strain field can be obtained; 3) the stability of the residual stresses can be improved. A practical example is used to demonstrate the effectiveness of the present method.

\section{THEORY}

The definition of an inverse problem for identifying the residual stresses in a homogeneous, linearly elastic and unloaded body, induced by welding can be stated as follows:

Equilibrium conditions: $\quad \partial \boldsymbol{\sigma}=\mathbf{0} \quad$ (in $V$ )

where $\sigma$ is residual stress and $V$ is the domain of the body.

Kinematics: $\quad \boldsymbol{\varepsilon}=\nabla^{s} \boldsymbol{u}$

where $\varepsilon$ denotes the strain in the body, and $\boldsymbol{u}$ is the displacement field.

Constitutive law:

$$
\sigma=\boldsymbol{D}\left(\varepsilon-\varepsilon^{*}\right)
$$

where $\boldsymbol{D}$ denotes the elastic matrix and $\varepsilon^{*}$ is the incompatible strain, i.e., the source of the residual stresses. Ueda and Fukuda [1] refers to $\varepsilon^{*}$ as the "inherent strain" in the body.

Boundary conditions: $\quad \boldsymbol{\sigma} \cdot \boldsymbol{n}=\mathbf{0}$ (on $\Gamma_{\sigma}$ ) and $\boldsymbol{u}=\overline{\boldsymbol{u}}$ (on $\Gamma_{u}$ )

where $\boldsymbol{n}$ is the normal direction of the surface and $\overline{\boldsymbol{u}}$ is the prescribed displacement vector.

Comparison with experimental results: $\quad \sigma_{i}=\bar{\sigma}_{i} \quad(i=1 \ldots k)$

where $\bar{\sigma}_{i}$ is the stresses at the experimental point $i$ obtained by experimental methods.

The task in this inverse problem is to find the inherent strain $\varepsilon^{*}$ with the aid of the experimental data, and use it to get the residual stresses. Naturally, for complicated bodies or domains, the FEM is employed to describe eqns (1)-(4). In terms of FEM, an inherent strain imposed onto an element is transformed into the equivalent elemental nodal forces via the local constitutive law and the local strain-displacement relation. After assembling the elemental load vectors constructed from the inherent strain and elemental stiffness matrices, the following equation can be obtained: $\boldsymbol{K} \boldsymbol{u}=\boldsymbol{F}$, where $\boldsymbol{K}, \boldsymbol{u}$ and $\boldsymbol{F}$ are the global stiffness matrix, the global displacement vector and load vector. Using the obtained $\boldsymbol{u}$, the strain field $\varepsilon$ can be computed using eqn (2). Finally the residual stresses can be evaluated from eqn (3).

First, the inherent strain field is described using the components of inherent strain at some points as follows,

$$
\varepsilon^{*}=\boldsymbol{g}(x, y, \boldsymbol{p})
$$


where $x, y$ and $\boldsymbol{p}$ are the coordinates and unknown components of inherent strain, respectively. From eqns (3) and (6), the unknowns in $\boldsymbol{p}$ are related to the stresses $\sigma^{F}$ at experimental points:

$$
\sigma^{F}=\boldsymbol{S p}
$$

where $S$ is the sensitivity matrix, which represents the change of the residual stress at the experimental points caused by unit change of the unknown parameters in $\boldsymbol{p}$.

Finally, the unknown parameters in $\boldsymbol{p}$ are determined by minimizing the difference between the experimental stresses and numerically calculated stresses in the least square sense: $\Phi=\left(\sigma^{F}-\bar{\sigma}\right)^{T}\left(\sigma^{F}-\bar{\sigma}\right) / 2$. For a welding plate in Figure 1, the method in [1] can be described as: 1) to discretize the plate using the FEM mesh in such a way that experimental points are coincident with the element nodes; 2) using the components of inherent strains at all mesh points in the plate as the unknown parameters $\boldsymbol{p}$ in eqn (6); 3) to construct the sensitivity matrix $\boldsymbol{S} ; 4$ ) to solve $\boldsymbol{p}$ from the least-square technique; 5) using the obtained inherent strains to get the residual stresses. The drawback of this method is that the large amount of experimental data is required since unknown parameters in $\boldsymbol{p}$ are too many. Also, this problem is usually ill-conditioned due to two reasons. The first is the singular modes in inherent strain. The inherent strains can be broken into two parts: effective inherent strain, which causes the residual stresses, and ineffective part (singular mode or compatible mode), which has no contribution to residual stresses and causes the problem ill-posed. The second one is the ill-conditioned sensitivity matrix, which can be attributed to the inappropriate locations of experimental points.

In this work, to increase stability of solution, the following strategis are advocated herein: 1) First, we use some polynomials of specific orders to approximate the inherent strain field. The unknown coefficients in the polynomials can be determined through some selected nodes (key-nodes) in the domain or on the boundary. To properly determine the order of the polynomials, the previous knowledge accumulated numerically or experimentally about the inherent strains and the residual stress should be considered; 2) After appropriately selecting the order of the polynomials, the singular modes in the inherent strain can be excluded. Due to the explicit form of the inherent strains in form of polynomial, some terms in the polynomials corresponding to the singular modes, which satisfy the compatibility condition explicitly, can be recognized and removed easily; 3) Thirdly, to get a stable result, the proper selection of locations of experimental points is accounted for. This selection can be rationalized using the 
singular value decomposition method (SVD) to analyze the sensitivity matrix $\boldsymbol{S}$. Then, we can adjust the positions of points to enhance the small singular values.

When using polynomials to simulate the inherent strain field, the computation of sensitivity matrix is different. First, the inherent strain field in form of polynomial is

$$
\boldsymbol{\varepsilon}^{*}=\boldsymbol{h}(x, y, \boldsymbol{a})
$$

where $\boldsymbol{a}$ is the vector composed by unknown coefficients of polynomials.

For the welding plate problem in Figure 1, a source area should be first assumed approximately. Out of this area, the inherent strain is thought of to be zero. This estimation can be obtained from the experiences and experimental data. Then, some key-nodes, i.e., the black points in Figure 1, should be selected in the source area. The inherent strains at these nodes are:

$$
\boldsymbol{p}=\left\{\varepsilon_{1}^{*}\left(x_{1}, y_{1}\right), \varepsilon_{2}^{*}\left(x_{2}, y_{2}\right), \ldots, \varepsilon_{n}^{*}\left(x_{n}, y_{n}\right)\right\}^{T}
$$

where $x_{i}, y_{i}$ and $\varepsilon_{i}^{*}$ are the coordinates, and the inherent strains of the key-node $i$.

When the number of components of inherent strain in the vector $\boldsymbol{p}$ are equal to the number of the unknown coefficients in the vector $\boldsymbol{a}$, the distribution of inherent strain field in eqn (8) can be determined easily if the $\boldsymbol{p}$ vector are known using the following equation,

$$
\boldsymbol{a}=\boldsymbol{L}(x, y, \boldsymbol{p})
$$

This is a one to one linear mapping process. For plane problems, two components of inherent strain at a key-node are assumed. Then, the sensitivity matrix can be calculated as follows: 1) $i=1$, in eqn (9), assume the first component of the inherent strain at the node $i$ equals to one and other components at other points are zero; 2) using assumed $\boldsymbol{p}$ and eqn (10), the unknown coefficients of polynomials for the inherent strain field, i.e., $\boldsymbol{a}$ can be determined. Then, from the distribution of inherent strain field in eqn (8), the elemental effective load vectors can be determined. Note that the part of inherent strain field out of the source area should be cut when computing the elemental effective load vectors as shown in Figure 1. Then, the global load vector can be formed; 3) from $\boldsymbol{K} \boldsymbol{u}=\boldsymbol{F}$, the displacements and the strain field can be calculated. Finally, from eqn (3), the residual stresses at the experimental points (white points), can be computed, which are the components of one column of the sensitivity matrix corresponding to the first component of inherent strain at the key-node $i$; 4) assume the second component of the inherent strain at the node $i$ equals to one and other components at other points are zero. Repeat 2) and 3) to compute the next column of the sensitivity matrix caused by the inherent strains at the node $i$; 5) $i=i+1$, go to 1 ) to calculate the other columns in $\boldsymbol{S}$ matrix. 


\section{A PRACTICAL EXAMPLE}

To verify this method, a steel plate with slit type butt welded joint [2] in Figure 2 is used. The welding conditions and material constants are given in Table 1, where $Q, t$ and $B$ are the heat input, plate thickness and welding length. Due to the symmetry of problem, only $1 / 4$ portion is modelled. The source area is: $0 \leq x \leq 350$ and $0 \leq y \leq 100$. The inherent strain fields are assumed as: $\varepsilon_{y}^{*}=a_{0} x+a_{1} x^{2}+a_{2} x^{3}+a_{3} x^{4}+a_{4} x y+a_{5} y x^{2}+a_{6} y x^{3}+a_{7} y x^{4}+a_{8} y^{2} x+a_{9} x^{2} y^{2}+a_{10} y^{2} x^{3}+a_{11} y^{2} x^{4}, \varepsilon_{x}^{*}=a_{12}+a_{13} y+$ $a_{14} y^{2}$ and $\gamma_{x y}^{*}=0$. In Table 2, 12 key-nodes, from which 12 components of $\varepsilon_{y}^{*}$ and 3 components of $\varepsilon_{x}^{*}$ are taken in $\boldsymbol{p}$, are shown. The number of components in $\boldsymbol{p}$ is equal to that of $a_{i}$ in the above polynomials for inherent strains. Also, 12 experimental points are selected in Table 2. There are 15 unknown parameters, and 24 components of residual stress at experimental points are used as experimental data. The results are given in Figures 3 and 4. We can find that this method can give a good estimation on the residual stresses for this practical problem.

\section{CONCLUSIONS}

A systematic strategy is proposed to estimate the residual stress caused by welding. The key idea is to introduce some polynomials to simulate the inherent strains. The singular modes can be excluded easily. The rational selection of the experimental points can be carried out through the SVD technique. Compared with the traditional inherent strain method [1], both the order of the

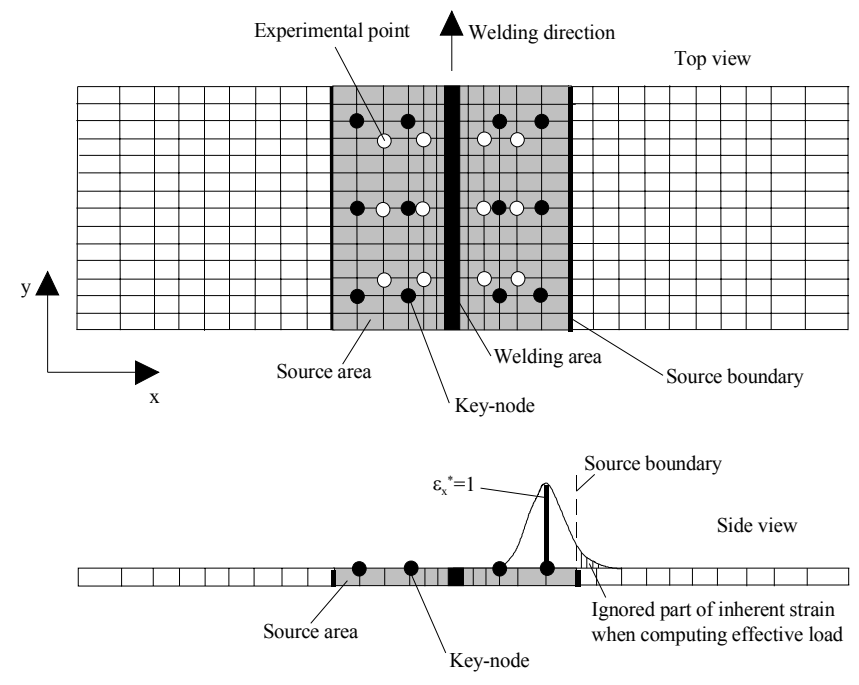

Figure 1: A welding plate with an estimated source area

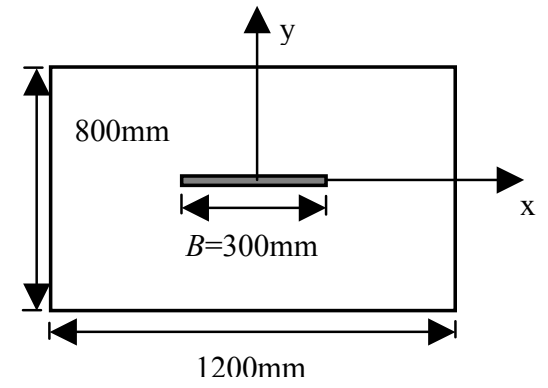

Figure 2: Practical welding of a plate 


\begin{tabular}{cc}
\hline$Q /(t B)$ & $4200 \mathrm{cal} / \mathrm{cm}^{2}$ \\
Young's modulus & $210000 \mathrm{MPa}$ \\
Poisson's ratio & 0.3 \\
Welding length & $300 \mathrm{~mm}$ \\
Yielding strength & $280 \mathrm{MPa}$ \\
\hline
\end{tabular}

\begin{tabular}{ccccc}
\hline & \multicolumn{2}{c}{$x$ coordinate $(\mathrm{mm})$} & \multicolumn{2}{c}{$y$ coordinate $(\mathrm{mm})$} \\
\cline { 2 - 5 } & $\begin{array}{l}\text { Key- } \\
\text { node }\end{array}$ & Exp. point & $\begin{array}{l}\text { Key- } \\
\text { node }\end{array}$ & Exp. point \\
\hline 1 & 10 & 0 & 0 & 0 \\
2 & 10 & 0 & 50 & 25 \\
3 & 10 & 0 & 100 & 75 \\
4 & 200 & 0 & 0 & 100 \\
5 & 200 & 0 & 50 & 175 \\
6 & 200 & 0 & 100 & 250 \\
7 & 300 & 50 & 0 & 0 \\
8 & 300 & 100 & 50 & 0 \\
9 & 300 & 150 & 100 & 0 \\
10 & 400 & 200 & 0 & 0 \\
11 & 400 & 250 & 50 & 0 \\
12 & 400 & 300 & 100 & 0 \\
\hline
\end{tabular}

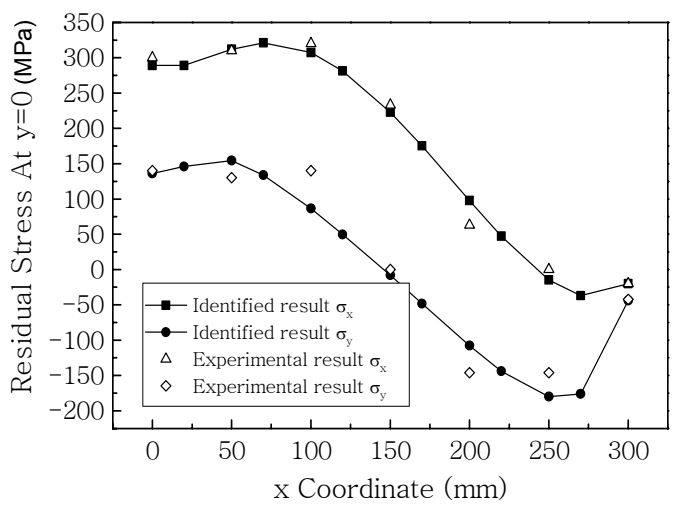

Figure 3: Identified and experimental results at $\mathrm{y}=0$

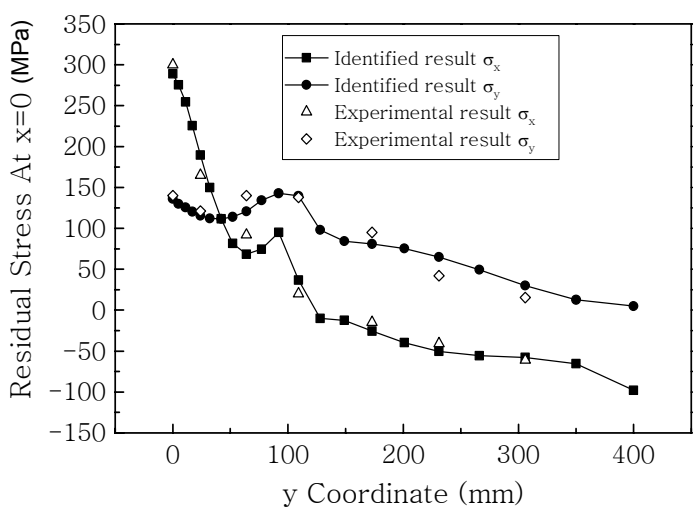

Figure 4: Identified and experimental results at $\mathrm{x}=0$

sensitivity matrix and experimental data can be reduced effectively. Furthermore, the stability of the results can be improved. It is shown in the example that this method can give an accurate and stable estimation on the residual stresses with small amount of experimental data.

\section{REFERENCES}

1. Ueda, Y. and Fukuda, K. New measuring method of three dimensional residual stress in long welded joints using inherent strain as parameters, ASME J. of Engineering Materials and Technology, 111, 1-8, 1989.

2. Fujimoto, T. Residual stresses in rectangular plates with slit-type butt welded joint, J. of the Japan Welding Society, 40, 111-125, 1971 (in Japanese). 